\title{
Effect of Feedback Data Rate in PIV Measurement-Integrated Simulation*
}

\author{
Takayuki YAMAGATA**, Toshiyuki HAYASE*** and Hiroshi HIGUCHI**** \\ ${ }^{* *}$ Graduate School of Engineering, Tohoku University \\ 6-6-01 Aramaki, Aoba-ku, Sendai, Miyagi, Japan \\ ***Institute of Fluid Science, Tohoku University \\ 2-1-1 Katahira, Aoba-ku, Sendai, Miyagi, Japan \\ E-mail: hayase@ifs.tohoku.ac.jp

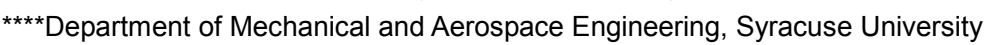 \\ 141 Link Hall, Syracuse, New York, U.S.A.
}

\begin{abstract}
This paper deals with measurement-integrated (MI) simulation, which is based on the observer in dynamical system theory to obtain the exact and detailed information of real flows. In this study, we propose the MI simulation coupled with Particle Image Velocimetry (PIV) for a flow with the Karman vortex street behind a square cylinder at Reynolds number of 1200 based on the cylinder width. The validity of the PIV-MI simulation is first examined in two-dimensional numerical experiment. The effect of feedback data rate is investigated by changing the feedback frequency and the feedback area. Then effectiveness of the PIV-MI simulation is validated in experiment. As a result, the PIV-MI simulation with full feedback shows good agreement with the standard solution for both the mean velocity and the velocity fluctuation. From the viewpoint of the reduced feedback data rate, spatially limited feedback shows better results than temporarily limited feedback. It is also confirmed that the information around the cylinder is important to reproduce the wake behind the cylinder. In the result of the experimental validation, the PIV-MI simulation reproduces the experimentally obtained flow field, showing the same effect of the feedback in accordance with the numerical experiment.
\end{abstract}

Key words: Measurement-Integrated Simulation, Hybrid Wind Tunnel, Flow Measurement, Particle Image Velocimetry, Karman Vortex, Flow Observer

\section{Introduction}

Both computational and experimental fluid dynamics are essential techniques in analysis of flow problems. However, numerical simulation and measurement have advantages and disadvantages from the viewpoint of reproducing "real flows" that contain complex boundary conditions and disturbances. Numerical simulation yields detailed information about a flow field, but its reliability depends on boundary and/or initial conditions and the degree of unknown disturbances. By measurement, on the other hand, information of an actual flow is obtained, but it is usually limited to partial information of the flow.

In order to obtain a solution for reproducing real flows, we have proposed measurement-integrated (MI) simulation ${ }^{(1)-(3)}$. The MI simulation is designed as a numerical simulation with the mechanism of feedback to include the information of a real flow from the measurement. The basic concept of this method is the observer in dynamical system theory. The MI simulation employs the numerical simulation instead of a linear differential 
equation as a model of the real flow. The availability of the MI simulation was shown in several studies. For example, the MI simulation was applied for numerical experiment for a developed turbulent flow in a square duct ${ }^{(1)}$, blood flow analysis by integrating ultrasonic measurement ${ }^{(2)}$ and analysis of the Karman vortex street behind a square cylinder on the hybrid wind tunnel ${ }^{(3)}$, which integrated a supercomputer and an experimental wind tunnel.

In this study, we consider the MI simulation of a flow past a square cylinder. This flow provides fundamental problems in many engineering applications. Many studies on the physical mechanics of wake flows and aerodynamic characteristics have been conducted by both numerical simulation and experiment ${ }^{(4)-(7)}$. Recently, large-scale computations such as LES of turbulent wake flow behind a square cylinder ${ }^{(8)}$ and simulation of three-dimensional vortical structures behind two square cylinders ${ }^{(9)}$ have been performed. Flow controls also have been conducted with a plate placed at upstream of a square cylinder ${ }^{(10)}$, and with a low-dimensional proper orthogonal decomposition (POD) for a wake behind a circular cylinder for the purpose of flow control ${ }^{(11)}$. The PIV velocity field and the surface pressure data over a stalled airfoil were correlated and the velocity field could be reconstructed using POD and Linear/Quadratic Statistical Estimates ${ }^{(12)}$. In the control area, especially, the information of the real flows is essential for practical application. In our previous research, the hybrid wind tunnel reproduced velocity and pressure fields of a real flow even with a coarse grid system for ordinary numerical simulation ${ }^{(13),(14)}$. The accuracy of the velocity field was validated by comparing to LDV measurement in time-averaged values, and the accuracy of the pressure field was investigated on the sidewall of the wind tunnel. In our previous studies, pressure measurements at the both sides of the cylinder were used as feedback signals, i.e. feedback points were only two to include the information of the real flow. Increase of the number of feedback points must be beneficial to reproduce the instantaneous flow structure of the real flow. For this purpose, PIV provides two or three components of velocity vectors in a measurement plane.

The idea of coupling numerical simulation and PIV or PTV is widely used to obtain pressure information or to improve the precision of the velocity measurement. For example, estimation of a pressure field by coupling Particle Tracking Velocimetry (PTV) and the Navier-Stokes equation ${ }^{(15)}$, unsteady flow simulation by combining PTV and $\mathrm{DNS}^{(16)}$. However, these studies mainly focus on improvement of measurement data with the aid of the numerical simulation. Therefore, reproducing the real flow by combining a limited number of measurement data and the numerical simulation is not performed. We speculate that the MI simulation is possible to reproduce the real flow in real time from the partial measurement. In the MI simulation, feedback data rate such as feedback frequency and feedback area is important in the reproduction of the real flow.

In this study, we propose Measurement-Integrated simulation using PIV. The validity of the PIV-MI simulation is first examined in two-dimensional numerical experiment. In the numerical experiment, a solution of a three-dimensional simulation with a fine grid system is used as a model of a real flow. The effect of feedback data rate is investigated by changing the feedback frequency and the feedback area. Then effectiveness of the PIV-MI simulation is validated in experiment.

\section{Problem Formulation}

In this study, we considered a flow past a square cylinder in a confined wind tunnel, in which the physical configuration was equivalent to that used in the former study of the hybrid wind tunnel ${ }^{(13),(14)}$. For numerical experiment of PIV-MI simulation, a standard solution was first obtained as a model of a real flow by a three-dimensional numerical simulation with a fine grid system. The PIV-MI simulation, on the other hand, was performed in two-dimensional formulation with a coarse grid system. It is assumed 
streamwise and wall-normal velocity components were obtained by PIV around the square cylinder as shown in Fig. 1.

\subsection{Governing equations}

Governing equations of general MI simulation are the Navier-Stokes equation with a body force term (1) and the continuity equation (2):

$$
\rho\left[\frac{\partial \mathbf{u}}{\partial t}+(\mathbf{u} \cdot \operatorname{grad}) \mathbf{u}\right]=-\operatorname{grad} P+\mu \nabla^{2} \mathbf{u}+\mathbf{f}
$$

$$
\operatorname{div} \mathbf{u}=0
$$

The difference of the MI simulation from ordinary numerical simulation is the existence of the artificial body force $\mathbf{f}$ corresponding to feedback signal as described later in this section. The governing equations were discretized with the finite volume method and they were solved with the algorithm similar to the SIMPLER method.

The PIV-MI simulation includes measured velocity as the feedback signals to compensate for the difference between the computation and the real flow. Feedback force $\mathbf{F}$ applied to a control volume is calculated in proportion to the difference between the measured velocity $\mathbf{u}_{m}$ and the computed one $\mathbf{u}_{\mathrm{c}}$ :

$$
\mathbf{F}=-K \cdot \Delta V\left(\mathbf{u}_{c}-\mathbf{u}_{m}\right) \frac{\rho U}{L}
$$

where $K$ is the feedback gain (non-dimensional), and $\Delta V$ is a control volume, and $L$ is a reference length (cylinder width $D$ ) and $U$ is a reference velocity. The flow in the control

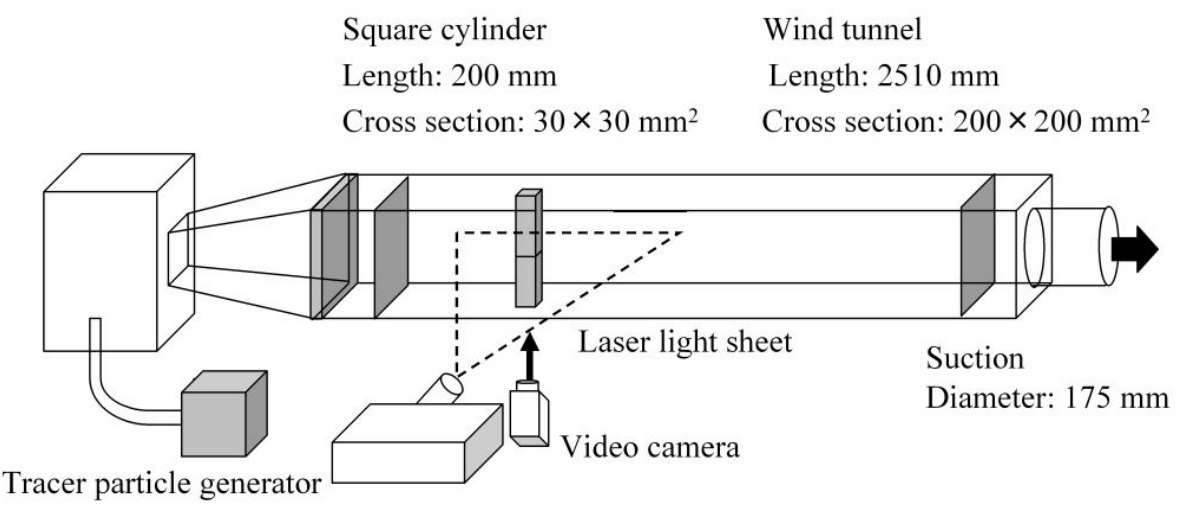

Fig.1 Experimental setup.

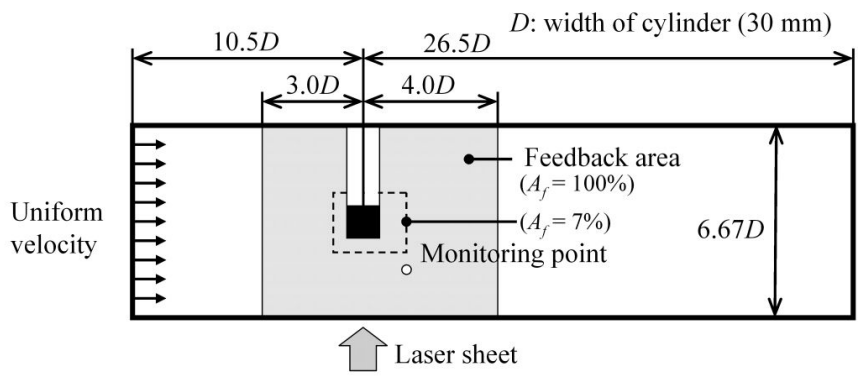

Fig. 2 Two-dimensional computational domain. 
Table 1 Feedback conditions.

\begin{tabular}{|c|l|l|l|l|}
\hline & $\begin{array}{l}\text { Feedback } \\
\text { data rate } \\
F_{d r}[1 / \mathrm{s}]\end{array}$ & $\begin{array}{l}\text { Feedback } \\
\text { area } A_{f}\end{array}$ & $\begin{array}{l}\text { Feedback } \\
\text { frequency } \\
F[\mathrm{~Hz}]\end{array}$ & $\begin{array}{l}\text { Feedback } \\
\text { gain } \\
{[-]}\end{array}$ \\
\hline Ordinary & 0 & $0(0 \%)$ & 0 & 0.0 \\
\hline & 393 & & 1 & \\
786 & & 2 & \\
Temporally & 1965 & & 5 & \\
limited & 2751 & $393(100 \%)$ & 7 & 3.0 \\
feedback & 3930 & & 20 & \\
& 7860 & & 50 & \\
& 19650 & & 100 & \\
\hline \multirow{5}{*}{ Spatially } & 39300 & $10.25 \%)$ & & \\
limited & 900 & $9(2 \%)$ & & \\
feedback & 2900 & $29(7 \%)$ & 100 & 3.0 \\
& 5700 & $57(15 \%)$ & 100 & \\
\hline
\end{tabular}

volume is accelerated or decelerated by the artificial forces to reduce the error of the velocity. Figure 2 shows the computational domain of the flow investigated herein. Two uniform staggered meshes were employed in this study: a two-dimensional grid system $(111 \times 21)$ for the PIV-MI simulation and a three-dimensional grid system $(444 \times 84 \times 84)$ for the standard solution. As the inflow boundary condition, a uniform velocity profile of the mean velocity $U_{0}=0.604 \mathrm{~m} / \mathrm{s}$ was specified corresponding to $R e=1200$ based on the cylinder width $D=0.03 \mathrm{~m}$, and the kinematic viscosity $v=1.512 \times 10^{-5} \mathrm{~m}^{2} / \mathrm{s}$. The time step was set at $\Delta t=0.01 \mathrm{~s}$ for both computations. Velocity components on the middle plane of the standard solution were first used for the PIV-MI simulation as the measured velocity. The effect of the feedback condition of an arbitrary data rate can be examined by using the standard solution instead of actual experimental data. The velocity components at the computational grid points were calculated with trilinear interpolation of those of the standard solution. The PIV-MI simulation with the actual PIV experimental data is discussed in later sections.

\subsection{Numerical experiment}

In order to evaluate the effect of the feedback data rate in PIV-MI simulation, numerical experiment was performed by changing the feedback frequency and the feedback area. The effects of these variations were compared on the mean velocity and the velocity fluctuation. Feedback conditions are summarized in Table 1. The full feedback area of $A_{f}=100 \%$ (see Fig. 2) was defined as a realistic area for PIV. There were 393 control volumes in the area on the coarse 2D grid system. The blank in the area represents the shadow of the laser sheet behind the square cylinder. The feedback areas less than $100 \%$ decrease the number of feedback points by a given factor by omitting the points far from the cylinder (see Fig. 2). The simulation with a feedback area $A_{f}=0 \%$ is equivalent to an ordinary numerical simulation without feedback. The vortex shedding frequency of the standard solution was $f_{s}$ $=3.03 \mathrm{~Hz}$ corresponding to the Strouhal number of $S_{t}=0.15$. Feedback frequency $F=100$ $\mathrm{Hz}$ means that the feedback acts every computational time step, and the other feedback frequencies mean intermittent feedback. The optimal feedback gain was determined as $K=$ 3.0 by test computations.

\subsection{Experimental validation}

Off-line PIV-MI simulation was performed using pre-measured actual experimental 
data to investigate the capability of the method. We first conducted the wind tunnel test using a PIV system in the wind tunnel shown in Fig. 1. The size of the cylinder is $200 \mathrm{~mm}$ in a span-wise length, $30 \times 30 \mathrm{~mm}^{2}$ in cross-section. The PIV system was set as shown in Fig. 1 to obtain the velocity field corresponding to the numerical experiment. The PIV system consists of a double-pulsed Nd: YaG laser (New Wave Research, Solo 120XT, energy: $120 \mathrm{~mJ} /$ pulse), a CCD camera (PCO, pco.1600, maximum frame rate: 30 frame/s, pixels: $1600 \times 1200)$, a timing controller (SEIKA, TT887) and a software for system control and image analysis (SEIKA, Koncerto). It is noted, however, that the actual frame rate was $14.8 \mathrm{~Hz}$ due to restriction of the camera. We seeded oil tracer particles of approximately 1 $\mu \mathrm{m}$ in the diameter into the wind tunnel with a seeding generator (PivTec, PivPart12). Images analyzed to generate steady statistics of flow field were about 350 pairs of images. The inflow velocity for the experiment was set at $0.604 \mathrm{~m} / \mathrm{s}$ corresponding to the Reynolds number of 1200 .

\section{Results and Discussion}

\subsection{Numerical experiment}

Computations of the flow around the square cylinder were carried out for various feedback conditions. Figure 3 compares time histories of velocity components at the monitoring point (see Fig. 2) among the standard solution, an ordinary simulation and PIV-MI simulations. Three typical results of the PIV-MI simulations are shown in the figure. The PIV-MI simulation with $A_{f}=100 \%$ and $F=100 \mathrm{~Hz}$ is of the feedback condition of maximum feedback data rate, while the other simulations correspond to the condition of

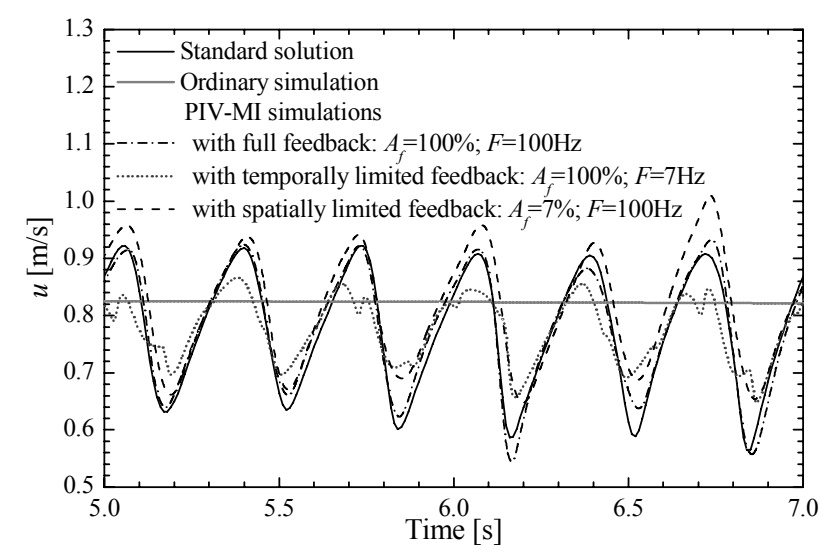

(a) Streamwise velocity

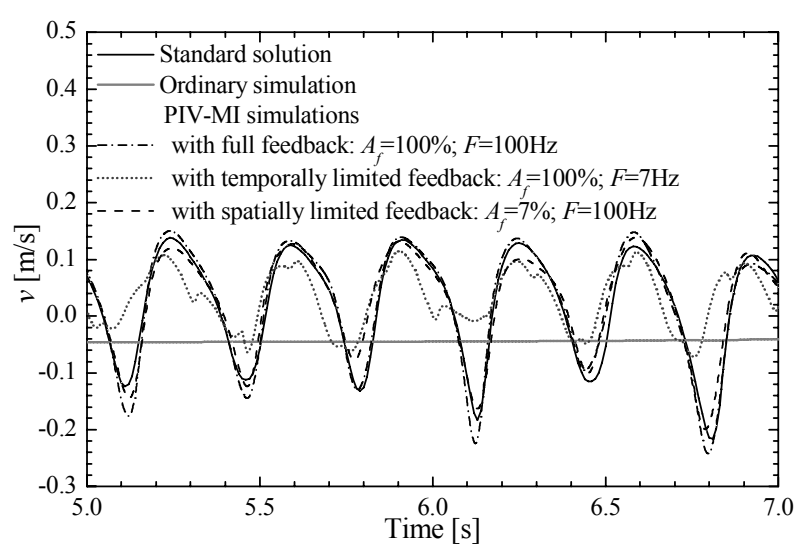

(b) Wall-normal velocity

Fig. 3 Time histories of velocity at the monitoring point. 

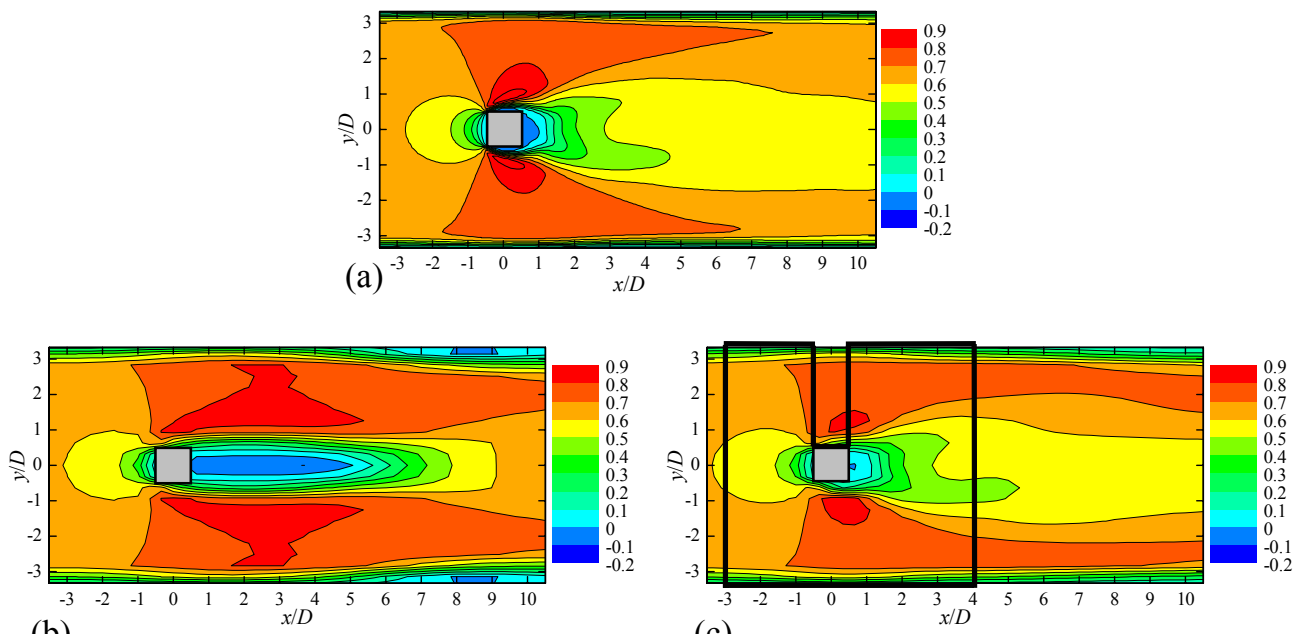

(b)

(c)
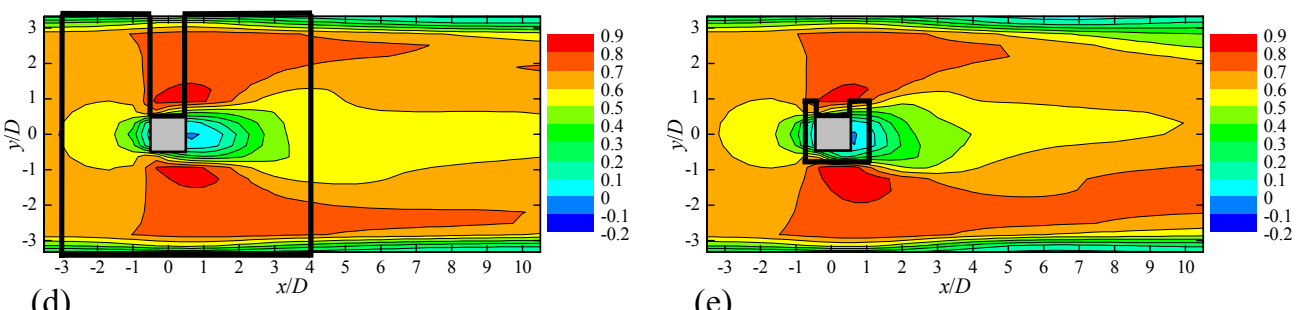

(e)

Fig. 4 Comparison of mean velocity $u_{m}$ : (a) standard solution; (b) ordinary simulation; (c) PIV-MI simulation with full feedback $\left(A_{f}=100 \%, F=100 \mathrm{~Hz}\right)$; (d) PIV-MI simulation with temporally limited feedback $\left(A_{f}=100 \%, F=7 \mathrm{~Hz}\right.$ ); (e) PIV-MI simulation with spatially limited feedback $\left(A_{f}=7 \%, F=100 \mathrm{~Hz}\right)$.

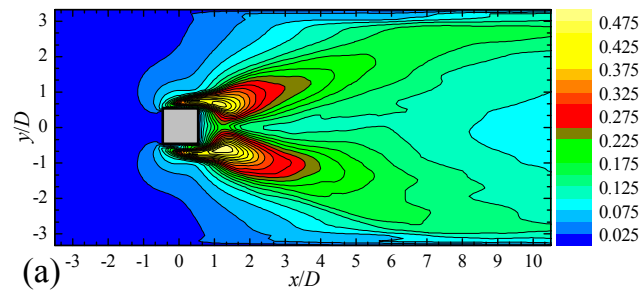

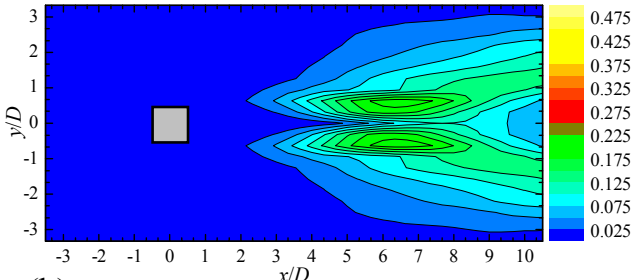

(b)

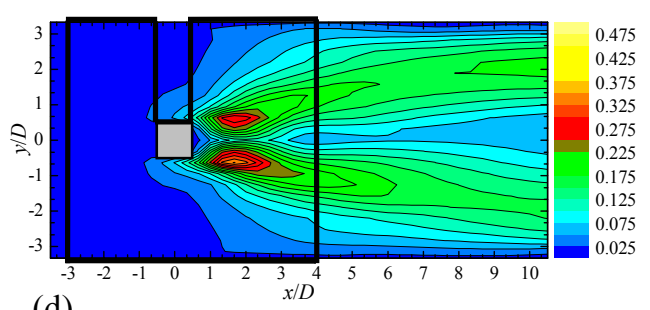

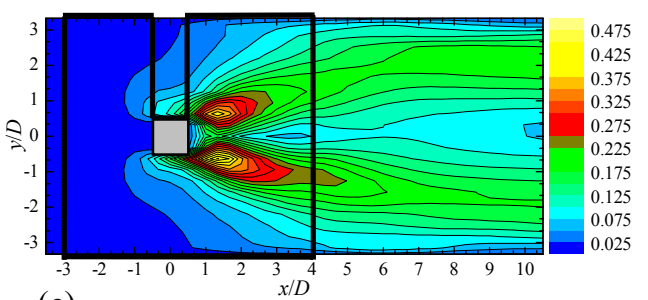

(c)

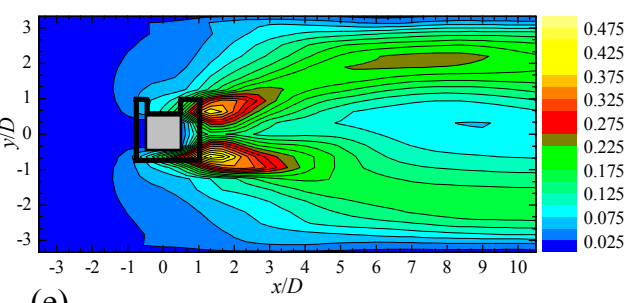

(e)

Fig. 5 Comparison of velocity fluctuations $u_{r m s}^{\prime}$ : (a) standard solution; (b) ordinary simulation; (c) PIV-MI simulation with full feedback $\left(A_{f}=100 \%, F=100 \mathrm{~Hz}\right)$; (d) PIV-MI simulation with temporally limited feedback $\left(A_{f}=100 \%, F=7 \mathrm{~Hz}\right)$; (e) PIV-MI simulation with spatially limited feedback $\left(A_{f}=7 \%, F=100 \mathrm{~Hz}\right)$. 


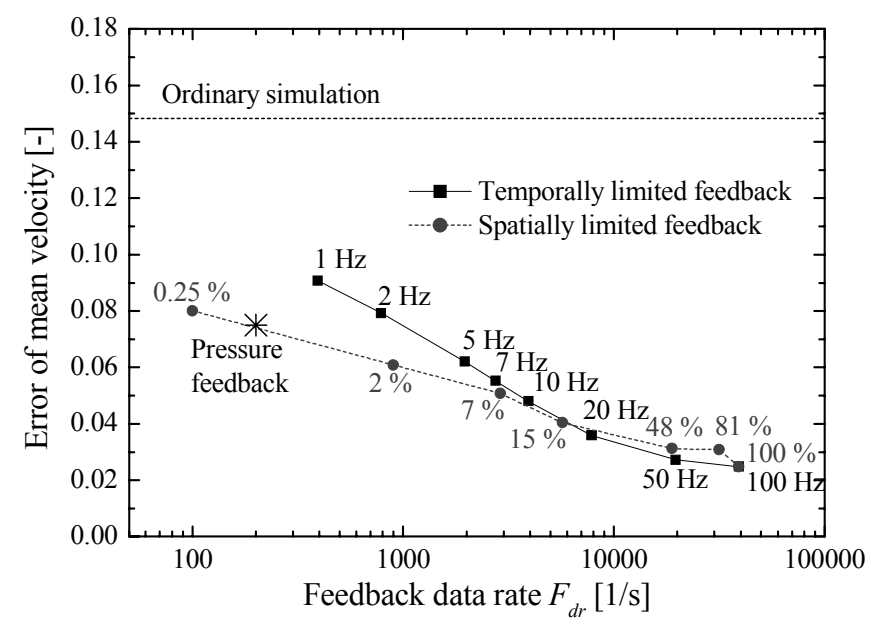

(a) Mean velocity $u_{m}$.

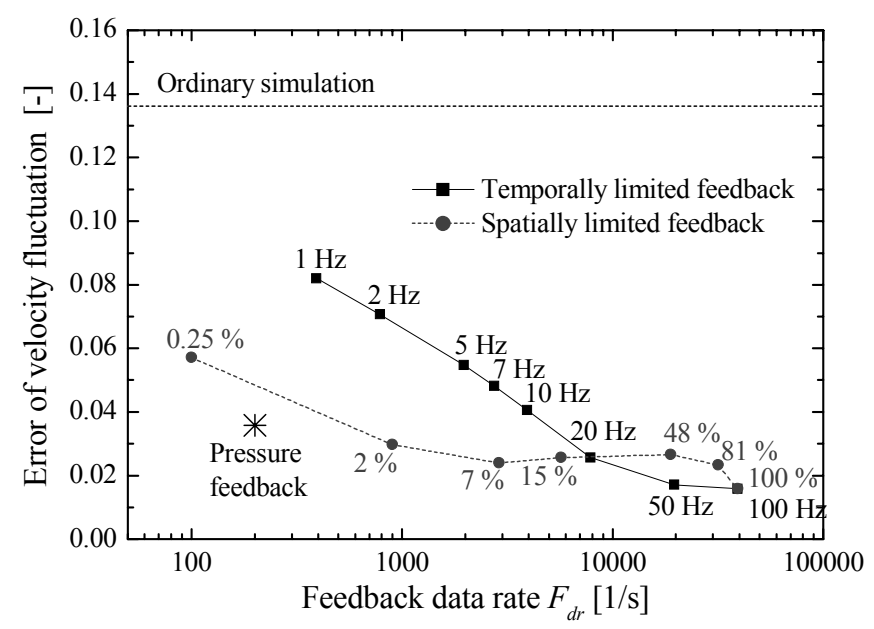

(b) Velocity fluctuation $u_{r m s}^{\prime}$.

Fig. 6 Mean error in streamwise velocity against feedback data rate.

the feedback data rate limited spatially or temporally. In Fig. 3(a), the streamwise velocity of the standard solution periodically oscillates due to the Karman vortex shedding. The ordinary simulation without feedback fails to reproduce unsteady flow oscillations because the grid system is too coarse to represent the vortex shedding properly. In contrast, the PIV-MI simulation of the full feedback data rate with $A_{f}=100 \%$ and $F=100 \mathrm{~Hz}$ properly converges to the standard solution. In addition, the PIV-MI simulation of spatially limited feedback data rate with $A_{f}=7 \%$ also shows good agreement with the standard solution, although the monitoring point is outside the feedback area. In the result of the PIV-MI simulation of the temporally limited feedback data rate with $F=7 \mathrm{~Hz}$, the velocity shows discontinuous changes at instants when intermittent feedback signals are applied, but it still shows good agreement with the standard solution in comparison with the ordinary simulation. Figure 3(b) shows the wall-normal velocity at the monitoring point, indicating results similar to the case of the streamwise velocity.

Distributions of the mean streamwise velocity are compared in Fig. 4 among (a) the standard solution, (b) ordinary simulation, (c) PIV-MI simulation of the full feedback data rate, (d) PIV-MI simulation of the temporally limited feedback data rate and (e) PIV-MI simulation of the spatially limited feedback data rate. The mean velocity is calculated for $5 \mathrm{~s}$ after a transient state. An extended recirculation region behind the cylinder in the result of 
the ordinary simulation (b) is improved in the PIV-MI simulation of the full feedback (c). By comparison between the PIV-MI simulations of temporally or spatially limited feedback (d) and (e), the difference is little for the mean velocity.

Figure 5 compares the root-mean-square values of the streamwise velocity fluctuation. The result of the standard solution in Fig. 5(a) shows large velocity fluctuations due to the Karman vortex shedding behind the square cylinder. In the result of the ordinary simulation (b), the peaks of the rms value are small and shift toward far downstream from the cylinder because the grid resolution is not enough (also see Fig. 3(a)). In contrast, the result of the PIV-MI simulation of the full feedback with $A_{f}=100 \%$ and $F=100 \mathrm{~Hz}$ (c) well reproduces the distribution of the standard solution. For the velocity fluctuations, the spatially limited feedback (e) shows a better result than the temporally limited feedback (d).

In order to evaluate the effect of feedback data rate, a mean error was calculated for the mean velocity and the velocity fluctuation in the area $-3.0 \leqq x / D \leqq 4.0$ including the whole feedback area and the shadow area. The mean error of the mean streamwise velocity is defined as

$$
\overline{e_{m}}=\frac{1}{U_{0}} \frac{1}{N} \sum_{i=1}^{N}\left|u_{c m}-u_{m m}\right| \text {, }
$$

where $U_{0}$ is the inflow velocity, $u_{c m}$ and $u_{m m}$ denote the mean velocity of the simulation and that of the standard solution respectively, $i$ denotes a grid index, $N$ is the number of grid points in the domain in which the error is evaluated. The mean error of the streamwise velocity fluctuation $u_{r m s}$ was calculated similarly.

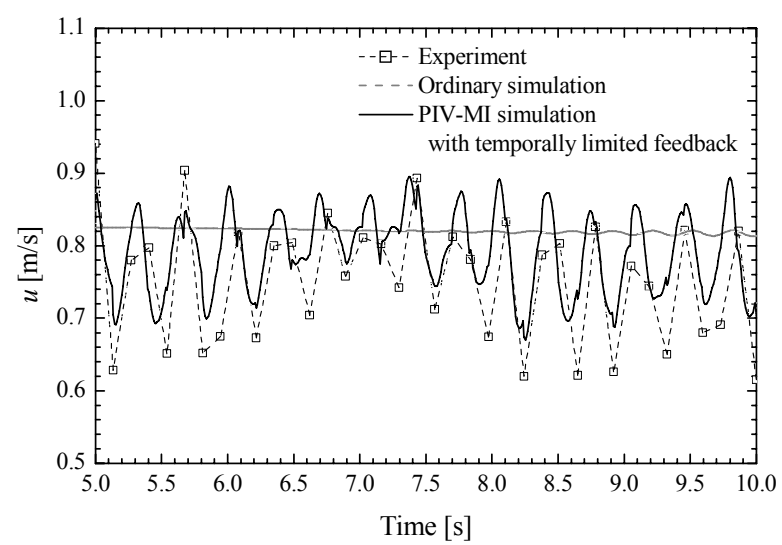

(a) Streamwise velocity

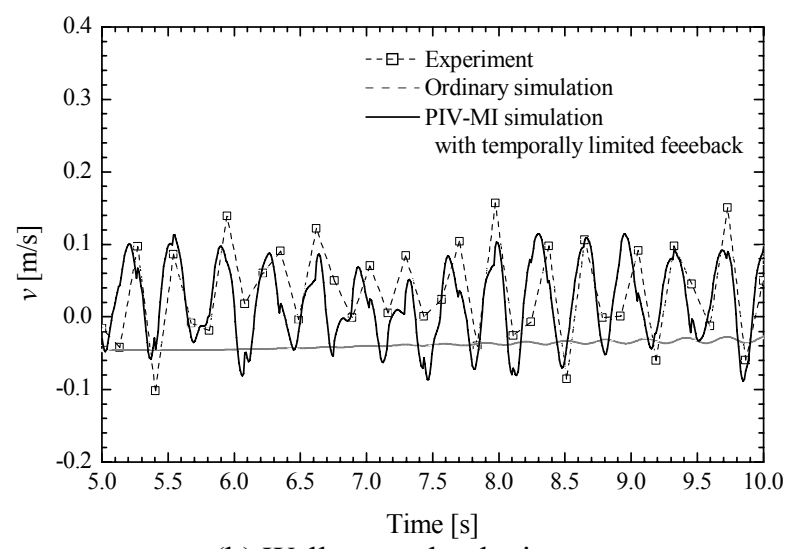

(b) Wall-normal velocity

Fig. 7 Experimental validation of PIV-MI simulation with time histories of velocity at the monitoring point. 
Figure 6 compares the errors of the mean velocity and the velocity fluctuation as a function of the feedback data rate $F_{d r}$ for temporally and spatially limited feedback. The error of the ordinary simulation is also plotted as a broken line for the purpose of comparison. The errors of the mean velocity are compared in Fig. 6(a). The nominal free stream velocity of the simulations is $U_{0}=0.604 \mathrm{~m} / \mathrm{s}$. The PIV-MI simulation of the full feedback with $A_{f}=$ $100 \%$ and $F=100 \mathrm{~Hz}$ substantially reduces the error in comparison with the ordinary simulation. The difference of the error between temporally and spatially limited feedback is little for the feedback data rate over $F_{d r}=3000 \mathrm{1} / \mathrm{s}$. In the figure, the result of former pressure feedback ${ }^{(14)}$ is also shown. The velocity feedback and the pressure feedback show almost the same effect for the mean velocity. Figure 6(b) compares the errors of the velocity fluctuation. In this figure, the PIV-MI simulation with spatially limited feedback is more effective for the velocity fluctuation than that with temporally limited feedback. It is notable that the error of the PIV-MI simulation with $A_{f}=0.25 \%$, i.e., with only one feedback point, decreases below half of the ordinary simulation. Comparing the results from the viewpoint of the feedback data rate, the feedback effectiveness is the same between the pressure information and the velocity information, and the information around the cylinder is relatively important to reproduce the Karman vortex street behind the cylinder.

\subsection{Experimental validation}

Next, PIV-MI simulation was performed using actual experimental data to investigate the validity of the proposed method. The computational time step was slightly changed from that of the previous section to $\Delta t=0.009 \mathrm{~s}$ to match the measurement sampling frequency. The feedback area was defined in almost the same area as in the previous numerical experiment except near the sidewall of the wind tunnel, because taking images was difficult in that area due to the restriction of the optical system. Feedback frequency was $F=7.4 \mathrm{~Hz}$, and the optimal feedback gain was determined as $K=3.0$ by test calculations.

Figure 7 compares time histories of velocity components at the monitoring point (see Fig. 2) among the experiment, the ordinary simulation and the PIV-MI simulation. Sampling frequency of the PIV measurement $(7.4 \mathrm{~Hz})$ is roughly twice of the frequency of the Karman vortex shedding $(2.94 \mathrm{~Hz})$. The velocity fluctuation of the PIV-MI simulation properly traces that of experiment in both streamwise and wall-normal directions.

Figure 8 compares the mean velocity (left) and the velocity fluctuation (right) of the streamwise velocity between the experiment (a) and the PIV-MI simulation (b). The data are

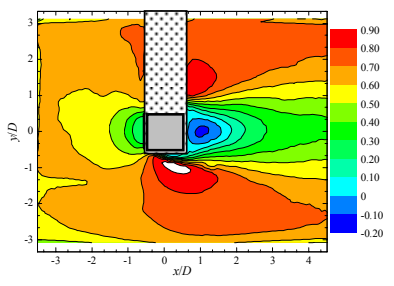

(a) Experiment
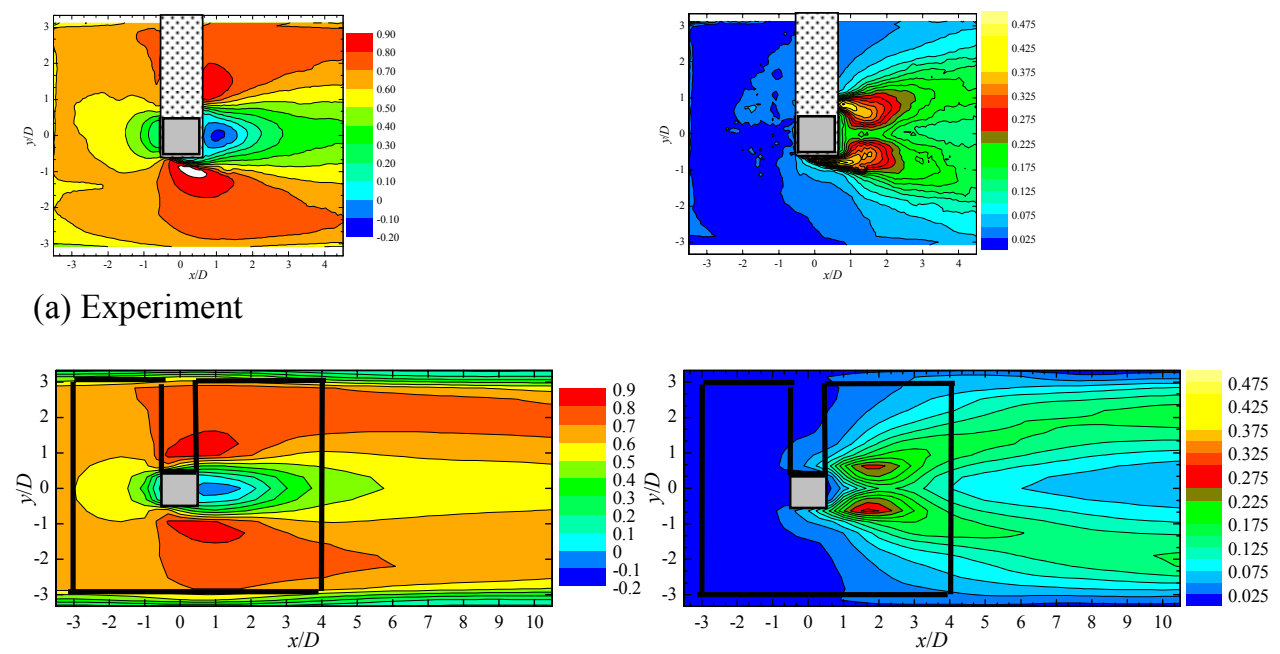

(b) PIV-MI simulation

Fig. 8 Experimental validation of PIV-MI simulation by comparison of streamwise velocity. Left: mean velocity; right: velocity fluctuation: (a) experiment; (b) PIV-MI simulation. 
absent in the shadow part of the cylinder where the laser light does not reach. In comparison with the standard solution for the previous numerical experiment, the region of reverse flow behind the square cylinder somewhat extends further downstream. In addition, the peak values of the velocity fluctuation are weaker than the standard solution. They are probably ascribed to the difference in inflow conditions. In the results of the PIV-MI simulation, improvement of the solution is seen in comparison with the ordinary simulation with the same boundary condition (Fig. 4(b) and Fig. 5(b)). In this study, it was difficult to perform the PIV measurement in the whole feedback domain in real time. But it was possible to perform two-dimensional simulation in real time with the present coarse grid ${ }^{(14)}$. Therefore, it is expected that real time PIV-MI simulation is possible by limiting the PIV measurement area around the cylinder.

\section{Conclusions}

In this study, PIV-MI simulation was performed for an unsteady flow past a square cylinder. The effect of the feedback data rate was evaluated in numerical experiment by temporally and spatially limiting the feedback data. The PIV-MI simulation with full feedback shows good agreement with a standard solution for both the mean velocity and the velocity fluctuation. From the viewpoint of the feedback data rate, spatially limited feedback and temporally limited feedback show similar performance for large feedback data rate, but the former feedback show better results for reduced feedback data rate. In addition, it is to be noted that the previous pressure feedback has an effect compatible to the present velocity feedback. It was also confirmed that the information around the cylinder was important to reproduce the wake behind the cylinder. The validity of the present PIV-MI simulation was confirmed experimentally. The PIV-MI simulation properly reproduced the experimentally obtained flow field in accordance with the numerical experiment. Possibility of real time PIV-MI simulation was also confirmed by using limited velocity information around the cylinder.

\section{Acknowledgement}

One of authors (TY) acknowledges the support of 2007 Global COE Program "Global Nano-Biomedical Engineering Education and Research Network Centre”. All computations were performed with the supercomputer system (Altix $3700 \mathrm{~B} \times 2$, SGI, Mountain View, CA, USA) in the Advanced Fluid Information Research Center, Institute of Fluid Science, Tohoku University.

\section{References}

(1) Hayase, T. and Hayashi, S., State Estimator of Flow as an Integrated Computational Method With the Feedback of Online Experimental Measurement, Journal of Fluids Engineering Transactions of the ASME, Vol. 119 (1997), pp. 814-822.

(2) Funamoto, K., Hayase, T. Shirai, A. Saijo, Y. and Yambe, T., Fundamental Study of Ultrasonic-Measurement-Integrated Simulation of Real Blood Flow in the Aorta, Annals of Biomedical Engineering, Vol. 33, No. 4 (2005), pp. 415-428.

(3) Nisugi, K., Hayase, T. and Shirai, A., Fundamental Study of Hybrid Wind Tunnel Integrating Numerical Simulation and Experiment in Analysis of Flow Field, JSME International Journal Series B, Vol. 47, No. 3 (2004), pp. 953-604.

(4) Davis R. W. and Moore E. F., A numerical study of vortex shedding from rectangle, Journal of Fluid Mechanics, Vol. 116, (1982), pp. 475-506.

(5) Okajima A., Strouhal numbers of rectangular cylinders, Journal of Fluid Mechanics, Vol. 123 
(1982), pp. 379-398.

(6) Davis R. W., Moore E. F. and Purtell L. P., A numerical-experimental study of confined flow around rectangular cylinders, Physics of Fluids, Vol. 27, No. 1 (1984), pp. 46-59.

(7) Nakagawa S., Nitta K. and Senda M., An experimental study on unsteady turbulent near wake of a rectangular cylinder in channel flow, Experiments in Fluids, Vol. 27 (1999), pp. 284-294.

(8) Kuroda M., Tamura T. and Suzuki M., Applicability of LES to the turbulent wake of a rectangular cylinder -Comparison with PIV data, Journal of Wind Engineering and Industrial Aerodynamics, Vol. 95 (2007), pp. 1242-1258.

(9) Sau A. and Hsu T. W., Three-dimensional evolution of vortical structures and associated flow bifurcations in the wake of two side-by-side square cylinders, Physics of Fluids, Vol. 19, 084105 (2007).

(10) Zhou L., Cheng M. and Hung K. C., Suppression of Fluid Force on a Square Cylinder by Flow Control, Journal of Fluids and Structures, Vol. 21 (2005), pp. 151-167.

(11) Cohen, K., Siegell, S. and McLaughlin, T., A heuristic approach to effective sensor placement for modeling of a cylinder wake, Computers \& Fluids, Vol. 35 (2006), pp. 103-120.

(12) Pinier, J. T., Ausseur, J., Glauser, M.N. and Higuchi, H., Proportional Closed-loop Feedback Control of Flow Separation, AIAA Journal, Vol. 45 No. 1 (2007), pp. 181-190.

(13) Hayase, T., Nisugi, K. and Shirai, A., Numerical Realization for Analysis of Real Flows by Integrating Computation and Measurement, International Journal for Numerical Methods in Fluids, Vol. 47 (2005), pp. 543-559.

(14) Yamagata T., Shibata H., Hayase T. and Smit K., Reproduction of a Real Flow with Karman Vortex Street by Integrating Flow Simulation and Pressure Measurement on an Obstacle, Proceedings of APCOM'07-EPMESC XI, (2007), CD-ROM.

(15) Murai Y., Nakada T., Suzuki T. and Yamamoto F., Particle tracking velocimetry applied to estimatie the pressure field around a Savonius turbine, Measurement Science and Technology, Vol. 18 (2007), pp. 2491-2503.

(16) Suzuki T., Mizushima T. and Yamamoto F., Hybrid Unsteady Flow Simulation Combining PTV and DNS at Moderate Reynolds Numbers, 37th AIAA Fluid Dynamics Conference and Exhibit, (2007). 